\title{
Familial Pseudohyperkalemia: A Rare Syndrome, But Diverse Genetic Heterogeneity
}

Key words: familial pseudohyperkalemia, hereditary stomatocytoses

The term familial pseudohyperkalemia (FP) was coined to describe an asymptomatic, dominantly inherited, red blood cell trait in which affected individuals present with high plasma $\mathrm{K}$ concentrations. The electrocardiogram is normal and no hyperkalemic conditions such as renal failure or Addison's disease are identified. The hematological abnormalities are minimal. It was determined that the rise in plasma $\mathrm{K}$ developed when the collected blood is allowed to stand at room temperature for some hours: plasma K estimations on fresh blood are normal. This syndrome was originally described by Stewart et al in 1979 (1). The proband, a 34-year-old Scottish woman was referred to them for her variable hyperkalemia. She had no particular abnormalities in both physical and laboratory examinations. However, when her blood was heparinized and incubated at room temperature, there were significant in vitro changes in the plasma $\mathrm{K}$ levels, suggesting the passive leakage of $\mathrm{K}$ from red blood cells. Interestingly, this phenomenon was not observed when the blood was maintained at $37^{\circ} \mathrm{C}$ in vitro. The pseudohyperkalemia in this family was attributable to a thermotropic abnormality in the so-called "passive leak" to $\mathrm{K}$ across the red blood cell membrane. This passive leak can conveniently be assessed experimentally as the residual $\mathrm{K}$ flux that persists in the presence of both ouabain and bumetanide (inhibiting the Na-K ATPase and Na-K-2Cl cotransporter). This original Scottish case is now referred as FP Edinburgh. Following the first description, other kindreds with pseudohyperkalemia due to temperature-dependent $\mathrm{K}$ loss from red blood cells in association with virtually normal hematology have been described (2-4).

To date, three types of pseudohyperkalemia devoid of hematological signs have been found based mostly on the abnormalities in the leak-temperature dependence curve. FP Edinburgh (1) and FP Lille (4) are characterized by a shallow slope abnormality in the interval $37^{\circ} \mathrm{C}$ to $20^{\circ} \mathrm{C}$. FP Cardiff (2) has the same $\mathrm{K}$ flux as normal at $37^{\circ} \mathrm{C}$, but shows a minimum at $23^{\circ} \mathrm{C}$ such that the flux at $0^{\circ} \mathrm{C}$ exceeded that at $37^{\circ} \mathrm{C}$ (a "U-shaped" abnormality). FP Chiswick and FP Falkirk (3) show a "shoulder" abnormality with a minimum at $25^{\circ} \mathrm{C}$, and a maximum at $10^{\circ} \mathrm{C}$, followed by a further fall.

In the current issue of the Journal, Sugimoto et al reported the first Japanese case of FP(5). This case, a 19-year-old girl, was referred to their hospital for occasional hyperkalemia without any physical and laboratory abnormal findings.

See also p 875 .

FP was diagnosed because of the marked difference in serum and plasma $\mathrm{K}$ level. The potassium leak from heparinized blood samples were demonstrated in vitro at $4^{\circ} \mathrm{C}$ and at room temperature, but not at $37^{\circ} \mathrm{C}$. The parents of the proband also showed the potassium leak at the lower temperature. According to their leak-temperature dependence curve, the proband will be classified as the Edinburgh and Lille type of FP.

Since kindreds with frankly hemolytic hereditary stomatocytoses such as dehydrated hereditary stomatocytosis (DHS) have been found to show similar temperature-related $\mathrm{K}$ effects to those found in FP (6-8) and the gene responsible for DHS has been mapped to 16q23-qter in a large Irish family (9), Iolascon et al performed the linkage analysis using microsatellite marker around the DHS locus (10). They found that the gene for FP Edinburgh mapped to the same arm of chromosome 16 as DHS, confirming that FP and DHS are most likely facets of the same genetically leaky red blood cell disease. Carella et al investigated the responsible locus for FP Lille in which cation fluxes are indistinguishable from those in FP Edinburgh (11). Microsatellite analysis excluded the 16q23-qter locus, and identified a novel locus mapped to 2q35-36. These findings suggest that FP Edinburgh and FP Lille, although they are phenocopies of one another, stem from two distinct loci, FP1 (16q23-qter) and $F P 2$ (2q35-36). This duality may suggest that the protein mediating the leak is a heterodimer.

It is puzzling that an extremely rare trait such as pseudohyperkalemia can still split into three genetic entities, and that the first of these entities can further split into two subentities. These clinical conditions demonstrate the genetic heterogeneity of FP. Further investigations are required to elucidate the responsible genes for the genetic heterogeneity of this rare syndrome.

Kenichiro KITAMURA, MD, PhD, FASN and Kimio TOMITA, MD, PhD

Department of Nephrology, Kumamoto University Graduate School of Medical Sciences, 1-1-1 Honjo, Kumamoto 860-8556 


\section{References}

1) Stewart GW, Corrall RJ, Fyffe JA, Stockdill G, Strong JA. Familial pseudohyperkalaemia. A new syndrome. Lancet 2: 175-177, 1979.

2) Gore DM, Chetty MC, Fisher J, Nicolaou A, Stewart GW. Familial pseudohyperkalaemia Cardiff: a mild version of cryohydrocytosis. Br J Haematol 117: 212-214, 2002.

3) Haines PG, Crawley C, Chetty MC, et al. Familial pseudohyperkalaemia Chiswick: a novel congenital thermotropic variant of $\mathrm{K}$ and $\mathrm{Na}$ transport across the human red cell membrane. Br J Haematol 112: 469-474, 2001.

4) Vantyghem MC, Dagher G, Doise B, et al. Pseudo-hyperkalemia. Apropos of a familial case. Ann Endocrinol (Paris) 52: 104-108, 1991 (in French).

5) Sugimoto T, Kume S, Osawa N, Nakazawa J, Koya D, Kashiwagi A. Familial pseudohyperkalemia: a rare cause of hyperkalemia. Intern Med 44: 875-878, 2005.

6) Coles SE, Ho MM, Chetty MC, Nicolaou A, Stewart GW. A variant of hereditary stomatocytosis with marked pseudohyperkalaemia. Br J Haematol 104: 275-283, 1999.

7) Entezami M, Becker R, Menssen HD, Marcinkowski M, Versmold HT. Xerocytosis with concomitant intrauterine ascites: first description and therapeutic approach. Blood 87: 5392-5393, 1996.

8) Grootenboer S, Schischmanoff PO, Cynober T, et al. A genetic syndrome associating dehydrated hereditary stomatocytosis, pseudohyperkalaemia and perinatal oedema. Br J Haematol 103: 383-386, 1998.

9) Carella M, Stewart G, Ajetunmobi JF, et al. Genomewide search for dehydrated hereditary stomatocytosis (hereditary xerocytosis): mapping of locus to chromosome 16 (16q23-qter). Am J Hum Genet 63: 810816, 1998.

10) Iolascon A, Stewart GW, Ajetunmobi JF, et al. Familial pseudohyperkalemia maps to the same locus as dehydrated hereditary stomatocytosis (hereditary xerocytosis). Blood 93: 3120-3123, 1999.

11) Carella M, d'Adamo AP, Grootenboer-Mignot $S$, et al. A second locus mapping to 2q35-36 for familial pseudohyperkalaemia. Eur J Hum Genet 12: 1073-1076, 2004. 